\title{
Modeling of Low-Capillary Number Segmented Flows in Microchannels Using OpenFOAM
}

\author{
D. A. Hoang ${ }^{*, \dagger}$, V. van Steijn ${ }^{\dagger}$, L. M. Portela ${ }^{* \dagger}$, M. T. Kreutzer ${ }^{\dagger}$ and C. R. Kleijn*,† \\ ${ }^{*}$ Multi-scale Physics Department, Delft University of Technology, Leeghwaterstraat 39, Delft, Netherlands \\ ${ }^{\dagger}$ Department of Chemical Engineering, Delft University of Technology, Julianalaan 136, Delft, Netherlands
}

\begin{abstract}
Modeling of low-Capillary number segmented flows in microchannels is important for the design of microfluidic devices. We present numerical validations of microfluidic flow simulations using the volume-of-fluid (VOF) method as implemented in OpenFOAM. Two benchmark cases were investigated to ensure the reliability of OpenFOAM in modeling complex physical phenomena in microfluidics, viz. 1) the steady motion of bubbles in capillaries, and 2) the formation of bubbles in T-junctions. We found that it is crucial to reduce spurious currents and to apply local grid refinement to capture the relevant flow physics. With these, we obtain good agreement between our numerical simulations and previously published theoretical and experimental data.
\end{abstract}

Keywords: CFD, OpenFOAM, microfluidics, bubbles

PACS: 47.11.Df, 47.55.db, 47.61.Jd

\section{INTRODUCTION}

Studies on segmented flows in microchannels are motivated by a wide range of applications $[1,2,3]$, such as the recovery of oil, the synthesis of microspheres for drug delivery, and the development of lab-on-a-chip devices, just to name a few. Instrumental in developing mechanistic insights of these flows is detailed time and spatially resolved data on e.g. velocities and pressures, which is difficult to obtain experimentally. Computational fluid dynamics (CFD) studies provide such data and allow broad parametric variations. Various numerical techniques have been used to study two-phase flows in microchannels.[4] Adaptive-grid methods [5, 6] are ideally suited to resolve the lubricating film between bubbles/droplets and channel walls, but they are complicated to apply to problems with large interface movement and topological change. Such problems are typically modeled by fixed-grid methods, including the Volume of Fluid [7, 8], level set [9], and diffuse interface method [10].

In this paper, we evaluate the capability of the volume of fluid method as implemented in the interFoam solver in OpenFOAM-dev1.6.x [11] for the modeling of segmented flows in microchannels. Two validation cases on the motion and the formation of bubbles are reported. Major challenges are 1) resolving the thin lubrication film surrounding the non-wetting bubbles to accurately capture the features of the flow, and 2) suppressing spurious currents arising from inaccurate calculations of the curvature of the interface. Our work demonstrates how to faithfully simulate these flows using OpenFOAM based on a comparison of our numerical results with experimentally-validated theory.

\section{NUMERICAL APPROACH}

In the Volume of Fluid method, a single set of Navier-Stokes equations for both phases is combined with an advection equation for the fluid fraction $\alpha$. We consider Newtonian fluids that are incompressible such that the governing equations can be written as:

$$
\begin{gathered}
\nabla \cdot \mathbf{U}=0 \\
\frac{\partial \rho \mathbf{U}}{\partial t}+\nabla \cdot(\rho \mathbf{U} \mathbf{U})=-\nabla p+\nabla \cdot \mu\left(\nabla \mathbf{U}+\nabla \mathbf{U}^{T}\right)+\rho \mathbf{f}+\mathbf{F}_{s} \\
\frac{\partial \alpha}{\partial t}+\nabla \cdot(\alpha \mathbf{U})-\nabla \cdot\left(\alpha(1-\alpha) \mathbf{U}_{r}\right)=0
\end{gathered}
$$

where $\mathbf{U}$ is the fluid velocity, $p$ the pressure, and $\mathbf{f}$ the gravitational force. The bulk density $\rho$ and viscosity $\mu$ are computed as the averages over the two phases, weighted based on the fluid fraction $\alpha . \mathbf{F}_{s}$ is the surface tension force, 
modeled as a volumetric force as described below.

In OpenFOAM, the fluid interface is sharpened by introducing the artificial compression term $\nabla \cdot\left(\alpha(1-\alpha) \mathbf{U}_{r}\right)$ in Eq. 3. The artificial compression velocity is given by $\mathbf{U}_{r}=\mathbf{n}_{f} \min \left[C_{\gamma}|\phi| /\left|S_{f}\right|, \max \left(|\phi| /\left|S_{f}\right|\right)\right]$, where $\mathbf{n}_{f}$ is the normal vector of the cell surface, $\phi$ is the mass flux, $S_{f}$ is the cell surface area and $C_{\gamma}$ is an adjustable coefficient which we set to 1 . It is only active in the interfacial region [12]. In the VOF method, the fluid fraction $\alpha$ is passively advected by the fluids. For incompressible flow, this is equivalent to a conservation law for fluid fraction, and therefore ensures the conservation of mass. The interfacial tension force is modeled as a body force by the Continuum Surface Force (CSF) method [13]. It is only active in the interfacial region and formulated as $\mathbf{F}_{s}=\sigma \kappa(\nabla \alpha)$, where $\sigma$ is the interfacial tension and $\kappa=\nabla \cdot(\nabla \alpha /|\nabla \alpha|)$ is the curvature of the interface.

A well-know problem in modeling low-Capillary number segmented flows is the appearance of spurious currents. We evaluated four methods to supress these currents by studying the relaxation of a droplet, that is the height function [14], the contour-based method [15], and two different smoothers [13, 16]. For the cases reported here, we found that the application of a smoother is the most efficient method with little difference between the two tested smoothers. The magnitude of spurious currents typically decreases three to four times compared to those produced in the original OpenFOAM code.

The PISO (pressure-implicit with splitting of operators) scheme is applied for pressure-velocity coupling [12]. We used a first order Euler scheme for the transient terms, controlling the time step by setting the maximum Courant number to 0.1. A first order Gauss linear scheme was used for the discretization of the gradient terms, and a second order TVD scheme for the divergence terms [12]. In all of our simulations, no-slip and zero contact angle boundary conditions were specified at the channel walls. A uniform velocity was applied at the inlet and an atmospheric pressure boundary condition and zero-gradient for fluid fraction $\alpha$ were applied at the outlet.

\section{RESULTS AND DISCUSSIONS}

\section{Motion of a two-dimensional bubble in a straight channel}

The first validation case considered here is the steady motion of a non-wetting air bubble in a straight twodimensional microchannel of width $w=100 \mu \mathrm{m}$. The bubble is dispersed in an immiscible liquid (ethanol), which separates the bubble from the walls by a thin lubricating film. For low-Capillary number flows, this film is typically two orders of magnitude thinner than the channel width, such that resolving the flow inside the film is computationally demanding. Combined with the fact that the features of the flow are well-known [17], this problem presents an excellent validation case.

To demonstrate how to successfully capture the features of the flow for capillary numbers between $10^{-4}$ and $10^{-2}$ and Reynolds numbers lower than 10, we applied different mesh sizes and mesh types and compared the film thickness $b$, the bubble velocity $U_{d}$, the pressure drop over the bubble $\Delta p$, and the shape of the interface $y(x)$ with the theoretical predictions by Bretherton [17]

$$
\begin{aligned}
& b / w=0.321\left(3 \mu U_{d} / \sigma\right)^{2 / 3} \\
& U_{d} / U_{c}=1+0.643\left(3 \mu U_{d} / \sigma\right)^{2 / 3} \\
& \Delta p w / \sigma=5.3\left(3 \mu U_{d} / \sigma\right)^{2 / 3} \\
& y / w=x^{2} / r^{2}+0.895\left(3 \mu U_{d} / \sigma\right)^{2 / 3}
\end{aligned}
$$

The influence of the uniform grid cell size, $\Delta$, on the thickness of the film is shown in Fig. 1a. All three grid sizes $(\Delta / w=0.014,0.01$, and 0.007$)$ give accurate predictions at relatively high values of the capillary number, which we defined based on the viscosity $\mu$ of the continuous phase and the velocity of the droplet $\left(\mathrm{Ca}=\mu U_{d} / \gamma\right)$. By contrast, none of these meshes resolves the lubricating film for $C a<5 \cdot 10^{-3}$. Consequently, the bubble velocity (Fig. 1b) and, to a lesser extend, the pressure drop (Fig. 1c) disagree with the theoretical predictions. To solve this problem without significantly increasing the number of grid cells, we applied local grid refinement. We hereby divided the cells close to the walls in four to five smaller cells as shown in the inset of Fig. 1d, while using the same base mesh elsewhere. This procedure ensures sufficient resolution in the liquid film region to accurately capture its thickness, which is one-third of the smallest base grid size $(\Delta / w=0.007)$ for the lowest value of the capillary number. We found that at least two grid cells are needed in the lubricating film to properly capture its thickness. Our results did not appreciably change for an even finer grid resolution. Refining the grid locally in the lubricating film hence allows the successful prediction 

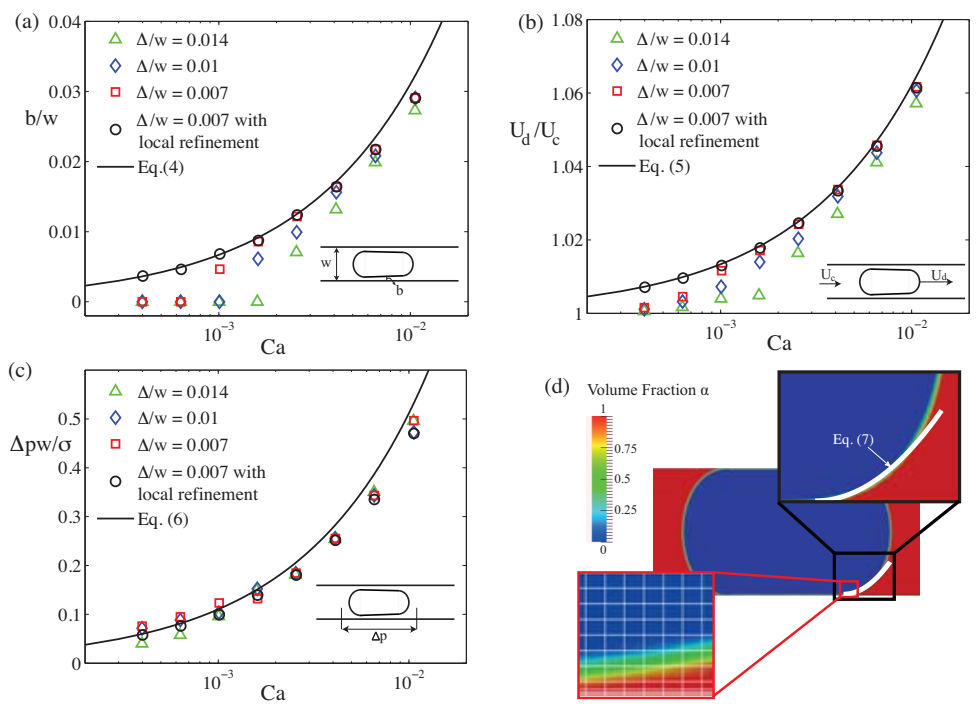

FIGURE 1. Validation of two-dimensional simulations on the steady motion of a non-wetting bubble through a $100 \mu \mathrm{m}$ wide microchannel based on a comparison of numerical simulations (symbols) and theory [17] (solid line). Dimensionless values of the (a) film thickness $b / w$, (b) bubble velocity $U_{d} / U_{c}$, and (c) pressure drop $\Delta p w / \sigma$ as a function of the capillary number for different mesh types and resolutions. (d) Comparison of the theoretical and numerical shape of a droplet for $\mathrm{Ca}=10^{-3}$ and base grid size $\Delta / w=0.007$ with local mesh refinement as depicted in the small inset. The numerical interface of a droplet is defined at $\alpha=0.5$.

of the thickness of the lubrication film as well as the other features of the flow as shown in Fig. 1a-d, without the need of using an extremely high resolution mesh elsewhere. Typical wall clock times for a simulation with the finest grid were 8 hours with a single processor. This is important, especially when extending two-dimensional simulations to three dimensions.

\section{Formation of three-dimensional bubbles in rectangular T-junctions}

In the previous section, we showed that the code is capable of simulating two-dimensional problems in which no large topological changes occur. For further validation, we evaluated a more complicated three-dimensional problem involving the breakup of a fluid interface, viz. the formation of bubbles in a microfluidic T-junction. Water and air were introduced from the main and side channel of the T-junction at flow rates $q_{c}$ and $q_{d}$, respectively, as shown in the inset of Fig. 2a. The volume of the formed bubble was computed for different ratios of flow rates and for different shapes of the T-junction with $h / w=0.11-0.33$ and $w_{i n} / w=0.67-3$. The Capillary number (here defined based on the viscosity and the velocity of the continuous phase $\left.C a=\mu_{c} U_{c} / \gamma\right)$ and the Reynolds number $\operatorname{Re}=\rho_{c} U_{c} h / \mu_{c}$ are on the order of $10^{-3}$ and $10^{-2}$, respectively, for all cases. Typical wall clock times for the simulation of one bubble formation period were 200 hours with 8 processors on a Beowulf Linux cluster.

To ensure reliable simulations, a grid-dependency study was performed. We computed the dimensionless bubble volume $V / h w^{2}$ for cell sizes ranging from $5 \mu \mathrm{m}(=w / 20)$ to $1.1 \mu \mathrm{m}(=w / 90)$ on a uniform mesh. For coarse meshes, the bubble volume strongly depends on cell size as shown in Fig. 2a. Grid-independent results were obtained for a total number of grid points $n=2 \cdot 10^{6}$, corresponding to a cell size of $\Delta=1.45 \mu \mathrm{m}$.

Based on this grid-dependency study, we studied the dependence of the dimensionless bubble volume $V / h w^{2}$ on the flow rate ratio $q_{d} / q_{c}$ for various T-junction geometries. Our simulations faithfully reproduce the linear dependence of the bubble volume on the flow rate ratio [18] as shown in Fig. 2b. Furthermore, our results closely agree with the results from VOF simulations for $w_{i n} / w=1$ by Chandorkar and Palit [8]. Moreover, our numerically predicted bubble volumes are in good quantitative agreement with predictions from the (experimentally-validated) analytical model by van Steijn and coworkers [19]. We note that for $w_{i n} / w=3$, it was necessary to apply local grid refinement at the walls to obtain good agreement. This ensured a difference of less than $7 \%$ between our CFD calculations and the theoretical model for all cases. 

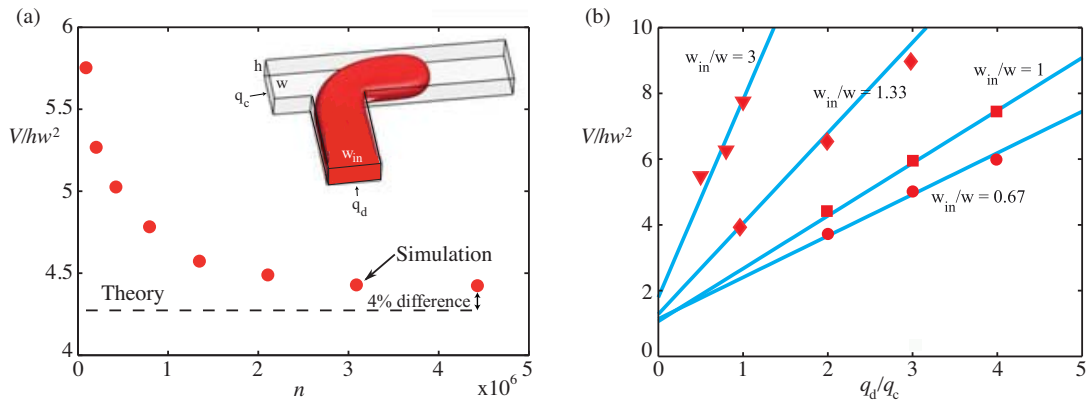

FIGURE 2. (a) Dependence of the dimensionless bubble volume $V / h w^{2}$ on the number of grid cells $n$. Geometry and conditions: $w=100 \mu \mathrm{m}, w_{i n} / w=1, h / w=0.33, q_{d}=10 \mu \mathrm{L} / \mathrm{min}, q_{c}=5 \mu \mathrm{L} / \mathrm{min}, C a=3.6 \cdot 10^{-4}$. (b) Dimensionless bubble volume $V / h w^{2}$ as a function of the ratio of flow rates for different T-junction geometries. The numerical data (symbols) agree well with theory [19] (solid lines). Geometries: $\left(w_{\text {in }} / w, h / w\right)=(3,0.33) ;\left(w_{\text {in }} / w, h / w\right)=(1.33,0.17) ;\left(w_{\text {in }} / w, h / w\right)=(1,0.33)$ and $\left(w_{\text {in }} / w, h / w\right)=$ $(0.67,0.11), q_{c}=1-5 \mu \mathrm{L} / \mathrm{min}$.

\section{CONCLUSION}

We presented two- and three-dimensional cases to validate the VOF method as implemented in OpenFOAM for lowCapillary number segmented flows in microchannels. Applying a smoother and local grid refinement is the key to reduce spurious currents and to capture the thin lubricating film between non-wetting bubbles/droplets and the channel walls. Based on a comparison between our results and theoretical models previously developed for the steady motion of bubbles in capillaries, we show that at least two grid cells are required in the lubricating film to model it. We also successfully captured physical details in a more complicated three-dimensional transient case, the formation of bubbles in T-junction. This paper shows that OpenFOAM can be used to accurately model low-Capillary number two-phase flows in microchannels.

\section{ACKNOWLEDGMENTS}

The authors gratefully acknowledge the financial support from STW and IROP-OSPT, the Netherlands.

\section{REFERENCES}

1. S. Y. Teh, R. Lin, L. H. Hung and A. P. Lee, Lab Chip 8, 198-220 (2008).

2. A. Huebner, S. Sharma, M. Srisa-Art, F. Hollfelder, J. B. Edel and A. J. Demello, Lab Chip 8, 1244-1254 (2008).

3. B. T. Kelly, J. C. Baret, V. Talyab and A. D. Griffiths, Chem. Commun. 18, 1773-1788 (2007).

4. M. Wörner, Microfluidic Nanofluidic, 1-46 (2012).

5. A. L. Hazel and M. Heil, J. Fluid Mech. 470, 91-114 (2002).

6. M. T. Kreutzer, F. Kapteijn, J. A. Moulijn, C. R. Kleijn and J. J. Heiszwolf, AlChE J. 51, 2428-2440 (2005).

7. D. Qian and A. Lawal, Chem. Eng. Sci. 61, 7609-7625 (2006).

8. A. Chandorkar and S. Palit, Nanotech 2009 Conference, edited by M. Laudon and B. Romanowicz,Nanotech Conference \& Expo 2009 Technical Proceedings 3, Houston, 2009, pp. 396-400.

9. M. Garzon, L. J. Gray and J. A. Sethian, J. Comput. Phys. 228, 6079-6106 (2009).

10. M. De Menech, P. Garstecki, F. Jousse and H. A. Stone, J. Fluid Mech. 595, 141-161 (2008).

11. H. G. Weller, G. Tabor, H. Jasak and C. Fureby, Comp. Phys. 12, 620-631 (1998).

12. H. Rusche, Ph.D thesis, Imperial College of Science, Technology and Medicine, 2002.

13. J. U. Brackbill, D. B. Kothe and C. Zemach, J. Comput. Phys. 100, 335-354 (1992).

14. S. J. Cummins, M. M. Francois and D. B. Kothe, Comput. Struct. 83, 425-434 (2005).

15. C. Kunkelmann, http://www.ttd.tu-darmstadt.de/forschung_1/openfoamdatenbank/bersicht. en.jsp.

16. B. Lafaurie, C. Nardone, R. Scardovelli, S. P. Zaleski and G. Zanetti, J. Comput. Phys. 113, 134-147 (1994).

17. F. P. Bretherton, J. Fluid Mech. 10, 166-188 (1961).

18. P. Garstecki, M. J. Fuerstman, H. A. Stone and G. M. Whitesides, Lab Chip 6, 437-446 (2006).

19. V. van Steijn, C. R. Kleijn and M. T. Kreutzer, Lab Chip 10, 2513-2518 (2010). 